and through the courtesy of the Boston City Hospital authorities and of Dr. Paul Thorndike, on whose service the patient was, the method was demonstrated by the writer. As this was the first use of intravenous anesthesia in this vicinity, and, so far as is known, in this country, a short description may be of interest to the profession.

Burkhardt usually employs three solutions:

(a) Physiological salt solution.

(b) Physiological salt solution containing 5\% ether.

(c) Physiological salt solution containing $1 \frac{1}{2} \%$ isopral.

Although in some cases the isopral solution was not used, the routine was to start the anesthesia with it and continue with the ether solution. These solutions are contained in large burettes, all connected by rubber tubing to a glass cannula, which is introduced into the vein in much the same manner as for the intravenous use of salvarsan. Any superficial vein may be used, but as a rule the median basilic of the left arm will be found the most convenient.

Anesthesia is induced somewhat more rapidly than by inhalation, and after it is complete the plain salt solution is alternated with that containing the ether according to the depth of anesthesia required. As a rule, less than half the amount of ether used by inhalation is necessary, and the patients recover with the customary unpleasant after effects of ether absent or much mitigated. The patient on whom it was demonstrated at the Boston City Hospital was a man fifty-one years of age, operated on for fistula in ano. He had a moderate degree of arteriosclerosis and was, therefore, not an ideal case for its use. No isopral was employed, the anesthesia being induced and maintained with the $5 \%$ ether solution, an occasional change being made to the plain salt solution. Sixteen hundred cubic centimeters of fluid in all were used in one-half hour, and of this $70 \mathrm{ccm}$. were ether. This is somewhat more than usual, but the excitement of the patient caused by the disturbances attending such a demonstration easily accounts for the excess. The anesthesia was satisfactory to the operator, Dr. Cotton, and the house-officer, Dr. Mahoney, reported a recovery with the usual after effects of ether much lessened in severity.

Intravenous anesthesia is indicated in operations about the head and neck, in hemorrhage, in weak cachetic patients, in diseases of the respiratory tract where inhalation anesthesia is objectionable, etc. It is contra-indicated in nephritis, extreme arteriosclerosis, severe heart lesions, plethoric patients, etc.

In the writer's opinion, its chief field of usefulness will be found in operations about the head and neck, where it is desirable to have the anesthetic and anesthetist removed from the surgical field, and here it would seem to be the method indicated in a majority of cases.

With reasonable care and skill in its administration the dangers from its use are only those inherent in ether itself. Any possible danger from air embolism is obviated if care is taken that the solution is running out of the cannula while it is being introduced into the vein, and that the flow is not interrupted during the anesthesia.

The writer has since used it at St. Elizabeth's Hospital, at the Massachusetts General Hospital, and hopes to make a more complete report of the method some time in the near future.

NOTE BY F. J. COTTON, M.D.

From the surgical point of view, the anesthesia was perfectly adequate in this case. It was rather curious to note the exact similarity of the anesthesia to that produced by the inhalation of ether. There was the same modification of respiration, the snoring as the patient went under, the dropping back of the tongue, and, when he was being brought into position for operation, there was the same stiffness of the legs, and the violent tremor on attempting to flex them, that are familiar accompaniments of ether anesthesia in these rectal cases. When the operation was finished, the recovery was somewhat more prompt than usual, and there was an entire freedom from nausea as well as vomiting. In this case, it is worthy of note, perhaps, that the stretching of the sphincter produced almost no reaction as shown in the breathing, less reaction than one usually sees in these cases. I suspect, however, that this was a variation in the individual reaction, and not characteristic of this method of administration.

I am inclined not only to agree with Dr. Dodge as to the rôle that this method is likely to play in the future, but should extend the field of its probable application to those cases also in which we particularly want to avoid the irritation of the lungs and throat from ether vapor, as in cases, for example, of bronchial pneumonia, phthisis or latent phthisis. There are certain of these cases that I think could probably be handled better in this way than even with the nitrous oxide-oxygen anesthesia. In throat cases, and cases of mouth surgery where we expect bleeding into the pharynx, the insufflation method (with a tracheal tube inserted through the mouth) will do better work, but in cases of surgery of the mouth and face, and even of the head, in which there is not going to be any bleeding in the mouth and throat, and in which the presence of the operator with his mask is a menace to asepsis and a great nuisance to the operator, this intravenous administration seems to me far the best method up to date.

\section{Clinital gDepartment.}

\section{A PARASITIC MULTILOCULAR OVARIAN CYST WEIGHING NINETY POUNDS.}

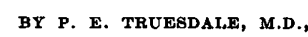

Surgeon to St. Anne's Hospital and the Highland Hospital, Fall River,

E. M., aged sixty-three. Nativity, Canada. Family history, negative. Past history, no pathological antecedents of importance. Had nine children; no puerperal accidents or difficulties. Menopause twelve 
years ago and uneventful. Patient had noticed an enlargement of the abdomen for many years. In 1906 she had acute abdominal symptoms. The pain and tenderness were referred to the sides of the upper abdomen. She remained in bed for about one week, and obtained relief by the application of mustard. No physician was called. From this time she observed a steady increase in the size of the abdomen. Her later discomforts consisted of an inability to walk or even maintain her equilibrium without assistance. She could lie only upon her right side. Other recumbent postures caused dyspnea, palpitation and thoracic pain. Body movements were attempted only by having a member of her household on either side to support the abdomen. Her appetite and digestion were fairly good. There had been constipation for several years, recently requiring brisk cathartics. Micturition was frequent without dysuria.

Physical examination. - The patient presented the typical picture of ovarian tumor; abdominal distention reaching to within a few inches of the knee, and the margin of the ribs spreading out like wings. There was edema of the lower extremities, with a marked varicose condition of the veins. Besides an appearance of general emaciation, she wore the characteristic facial expression.

Measurements of the abdomen were as follows: Ensiform to pubes, $32 \frac{1}{2}$ inches; greatest circumference, 55 inches.

Weight of the patient upon admission, $219 \mathrm{lb}$. When discharged on Jan. 31, 1911, she weighed $129 \mathrm{lb}$. Size of the tumor estimated at $90 \mathrm{lb}$.

On Jan. 5 the tumor was tapped and $25 \mathrm{lb}$. of clear viscid fluid were withdrawn. On Jan. 6 the tumor was removed.

The features of interest in the progress of the operation were: (1) The adhesions of the omentum through which large vessels communicated with the upper portion of the tumor. (2) Vascular adhesions to the peritoneum on the under side of the costal arch extending upward to the diaphragm. (3) A pedicle about two inches in length, no larger than the little finger, rotated about half a circle and having its origin in the right broad ligament.

The hemorrhage following the separation of the adhesions from the parietal peritoneum was quite troublesome. Control was brought about by the use of large pads wrung out of hot sterile water. The abdomen was then filled with about five gallons of waier and closed.

A good recovery was made. Less discomfort was experienced than is usual after laparotomy. The water was very slowly absorbed by the peritoneum. Even after ten days there was free fluid in moderate amount.

The patient was discharged from the hospital in good condition on Jan. 31, 1911.

Pathological examination of the tumor was made by Dr. Mary W. Marvell with the report as follows: Specimen is a sac of an ovarian cyst. Measures $40 \mathrm{~cm}$. $\mathrm{x} 28 \mathrm{~cm} . \mathrm{x} 16.5 \mathrm{~cm}$. and weighs $17 \mathrm{lb}$. Section shows the tumor to be made up of many cysts of varying sizes, the contents of which were gelatinous and in color varying from a clear straw to a dirty brown.

Microscopical section shows a mass of small cysts, lined by a single layer of cylindrical epithelium, supported by a very cellular connective tissue in which are numerous blood vessels. The epithelium in some of the cysts is arranged into finger-like processes. Diagnosis, multilocular ovarian cystadenoma.

The history of acute symptoms in 1906 was probably due to a partial rotation of the pedicle with degenerative and inflammatory changes at the fundus resulting in the formation of adhesions and a collateral blood supply.

A remarkable case of this sort is reported by Kelly (vol. ii, p. 250), in the illustration of which is shown the atrophied pedicle and the cyst extensively adherent to the posterior peritoneum, mesentery, colon and omentum.

Smitr, in 1906, reported a case before The Massachusetts Medical Society in which the tumor removed weighed $192 \mathrm{lb}$. and the diameter of the pedicle measured not more than $\frac{1}{2}$ inch. He found extensive adhesions to the entire front of the abdomen covering a space of about 3 square feet extending to the diaphragm, and concluded that the adhesions furnished most of the blood supply. His patient had been treated by electricity and osteopathy. To these forms of treatment Dr. Smith attributed the vascular adhesions which he encountered. The adhesions in this case, though aggravated by mechanical treatment, were originally formed more probably as a result of an interference with nutrition through the pedicle.

The frequency with which we find ovarian cysts with twisted pedicles emphasizes the tendency of these smooth and elastic tumors to rotate. There can be all degrees of rotation, from a slight twist which corrects itself to a complete strangulation.

A partial rotation of the pedicle interferes markedly with the blood supply and the more remote parts of the tumor break down and undergo inflammatory reaction, become adherent to all the contiguous parts of the peritoneum, and the vascular adhesions thus formed determine its parasitic existence.

In the case reported above the rapid growth of the tumor appeared to follow the period when a collateral circulation was established.

In my case, and in that of Dr. Smith, the parts of the tumor most remote from the pedicle became attached in the above manner.

Tumors of this size are now only rarely seen, whereas their removal was reported quite frequently in the literature of Dr. Homans's period. Cysts of this parasitic order were, no doubt, removed with great risk, inasmuch as there was considerable exposure of the peritoneum to sepsis and much hazard in the separation of the old vascular adhesions.

\section{Aledital Hrogregg.}

\section{REPORT ON PEDIATRICS.}

BY ThOMAs MORgaN ROTCH, M.D., AND CHARLES HUNTER DUNN, M.D., BOSTON.

\section{SCARLET FEVER.}

A publication by A. E. Vipond ${ }^{1}$ has announced the finding of what the writer believes to be the specific organism of scarlet fever. He first came to the conclusion that the specific organism would probably be found in the lymph nodes, and confined his work entirely to the glandular system. From seven consecutive cases 